\title{
Pharmacokinetics of S-1 in patients with peritoneal dissemination of gastric cancer
}

\author{
TAKASHI OSHIMA, ROPPEI YAMADA, SHINSUKE HATORI, CHIKARA KUNISAKI and TOSHIO IMADA
}

Gastroenterological Center, Yokohama City University Medical Center, Yokohama, Japan

Received December 27, 2005; Accepted February 16, 2006

\begin{abstract}
The response of gastric cancer with peritoneal dissemination to systemic chemotherapy may be negatively affected by poor drug delivery due to the blood-peritoneal barrier. However, S-1 has been reported to be effective. We examined the pharmacokinetics of S-1 in 14 patients who had gastric cancer with peritoneal dissemination. S-1 was given from the morning of the day before surgery to the morning of surgery. Concentrations of 5-fluorouracil (5-FU) and gimeracil (CDHP) were measured in the serum, ascites, disseminated peritoneal nodes, and normal peritoneum. There was a strong correlation between 5-FU and CDHP concentrations in peritoneal tissues. The concentrations of 5-FU and CDHP in the serum were similar to those in ascites. The concentration of 5-FU was significantly higher in disseminated nodes than in the normal peritoneum. After administration of S-1 to gastric cancer patients with peritoneal dissemination, 5-FU and CDHP in the serum linearly pass through the peritoneum and enter the ascites. High concentrations of 5-FU selectively penetrate disseminated peritoneal cells.
\end{abstract}

\section{Introduction}

Peritoneal dissemination is the most frequent cause of death from gastric cancer (1). Although systemic chemotherapy (2-5), intraperitoneal chemotherapy (6-8), and thermochemotherapy (9-12) have been used to manage peritoneal dissemination, the response rate has fallen short of expectations. A poor response rate has been attributed to the inadequate passage of systemically administered anticancer drugs to peritoneal tissues (13) and the prompt absorption of intraperitoneally administered drugs from the peritoneum, resulting in low drug concentrations in the peritoneal cavity (14).

S-1 was developed as an oral anticancer drug for the treatment of gastric cancer. S-1 consists of tegafur (a prodrug

Correspondence to: Dr Takashi Oshima, Gastroenterological Center, Yokohama City University Medical Center, 4-57 Urafune-cho, Minami-ku, Yokohama 232-0024, Japan

E-mail: ohshimatakashi@yahoo.co.jp

Key words: chemotherapy, gastric cancer, gimeracil, peritoneal dissemination, S-1, 5-fluorouracil of 5-FU), CDHP (an inhibitor of 5-FU metabolizing enzymes), and oteracil potassium, which specifically reduces gastrointestinal toxicity due to 5-FU, in a molar ratio of 1.0:0.4:1.0 (15). S-1 has proved to be clinically effective (16-18). Studies have demonstrated that S-1 maintains the quality of life of patients who have gastric cancer with peritoneal dissemination (19-22). The response of peritoneal dissemination from gastric cancer to systemic chemotherapy may be negatively affected by poor drug delivery due to the intraperitoneal bloodperitoneal barrier (13). Available evidence indicates that S-1 is more effective than conventional chemotherapy against peritoneal dissemination. Experiments using a model of peritoneal dissemination from gastric cancer in nude mice have shown that $\mathrm{S}-1$ penetrates the peritoneum and prolongs survival $(23,24)$. We undertook this study to determine whether $\mathrm{S}-1$ penetrates disseminated peritoneal nodes in patients with gastric cancer.

\section{Materials and methods}

Patients. We studied 14 adults younger than 75 years who had no complications and underwent surgery for a preoperative diagnosis of advanced gastric cancer invading the serosa (T3) or deeper by Japanese Classification of Gastric Carcinoma (25) between April 2003 and March 2004 (Table I). The study protocol was reviewed and approved by the Medical Ethics Committee of the Yokohama City University, School of Medicine. Fully informed consent was obtained from all patients participating in the study.

Sample collection. S-1 was given in a dose of 80-120 mg/day in two divided doses, calculated on the basis of body surface area, on the day before surgery and about $3.5 \mathrm{~h}$ before the scheduled time of surgery. During surgery, samples of the serum, ascites, disseminated parietal peritoneal nodes, disseminated mesenteric nodes, normal parietal peritoneum, normal mesentery, and greater omentum were obtained, approximately $4 \mathrm{~h}$ after the administration of S-1. In patients who underwent gastrectomy, samples of gastric cancer tissue and normal gastric mucosa were obtained. All specimens were stored at $-80^{\circ} \mathrm{C}$ immediately after collection.

Measurement of 5-fluorouracil (5-FU) and gimeracil (CDHP). Tissues were homogenized in 3 volumes of ice-cold saline and centrifuged at $10000 \mathrm{x} \mathrm{g}$ for $20 \mathrm{~min}$. The obtained supernatants were considered crude extracts containing 5-FU and 
Table I. Patient characteristics by Japanese Classification of Gastric Carcinoma.

\begin{tabular}{|c|c|c|c|c|c|c|c|}
\hline \multirow{2}{*}{$\frac{\text { Patient }}{1}$} & \multirow{2}{*}{$\begin{array}{c}\text { Age/ } \\
\text { Gender }\end{array}$} & & \multicolumn{4}{|c|}{ Microscopic and surgical findings } & \multirow{2}{*}{$\begin{array}{c}\begin{array}{c}\text { Conclusive } \\
\text { stage }\end{array} \\
\text { IIIb }\end{array}$} \\
\hline & & $\mathrm{t} 3$ & $\mathrm{n} 2$ & $\mathrm{P} 0$ & H0 & M0 & \\
\hline 2 & $59 \mathrm{M}$ & $\mathrm{t} 3$ & n3 & $\mathrm{P} 1$ & HO & M0 & IV \\
\hline 3 & $66 \mathrm{M}$ & $\mathrm{t} 4$ & $\mathrm{n} 1$ & P0 & HO & M0 & IV \\
\hline 4 & $73 \mathrm{~F}$ & $\mathrm{~T} 4$ & N3 & $\mathrm{P} 1$ & HO & M0 & IV \\
\hline 5 & $55 \mathrm{M}$ & $\mathrm{t} 3$ & n3 & P0 & $\mathrm{HO}$ & M1 & IV \\
\hline 6 & $72 \mathrm{M}$ & $\mathrm{t} 3$ & n1 & P0 & HO & M0 & IIIa \\
\hline 7 & $68 \mathrm{~F}$ & $\mathrm{t} 3$ & n1 & P0 & HO & M0 & IIIa \\
\hline 8 & $75 \mathrm{M}$ & $\mathrm{t} 3$ & n2 & P0 & HO & M0 & IIIb \\
\hline 9 & $62 \mathrm{~F}$ & $\mathrm{t} 3$ & n0 & P0 & HO & M0 & IIIa \\
\hline 10 & $72 \mathrm{M}$ & $\mathrm{t} 3$ & $\mathrm{n} 2$ & P0 & H0 & M0 & IIIb \\
\hline 11 & $70 \mathrm{M}$ & $\mathrm{t} 4$ & $\mathrm{n} 2$ & P0 & HO & M0 & IV \\
\hline 12 & $74 \mathrm{M}$ & $\mathrm{t} 3$ & n3 & P1 & $\mathrm{H} 0$ & M0 & IV \\
\hline 13 & $58 \mathrm{M}$ & $\mathrm{t} 3$ & n2 & P0 & HO & M0 & IIIb \\
\hline 14 & $45 \mathrm{M}$ & $\mathrm{t} 3$ & $\mathrm{n} 2$ & P0 & H0 & M0 & IIIb \\
\hline
\end{tabular}

(a)
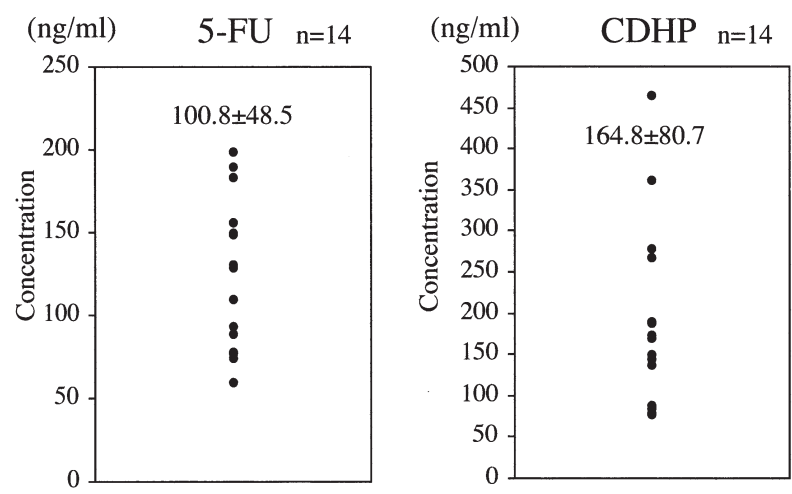

Figure 1. Concentrations of 5-FU and CDHP in serum. Serum concentrations of 5-fluorouracil (a) and CDHP (b) were 100.8 448.5 and $164.8 \pm 80.7 \mathrm{ng} / \mathrm{ml}$, respectively, $4 \mathrm{~h}$ after oral administration of S-1. 5-FU, 5-fluorouracil; CDHP, gimeracil.

CDHP. To $0.1 \mathrm{ml}$ of known amounts of the internal standard (ID) solution, $1 \mathrm{ml}$ of the serum, ascites or crude extracts was added, and the mixture was shaken with $5 \mathrm{ml}$ of chloroform. The mixture was then centrifuged at $3000 \mathrm{rpm}$ for $10 \mathrm{~min}$, and the organic layer was removed. The aqueous layer containing 5-FU or CDHP was treated twice with $4 \mathrm{ml}$ of ethyl acetate, and the two organic extracts were combined and evaporated at $50^{\circ} \mathrm{C}$ under a gentle stream of nitrogen gas. The residue was dissolved in distilled water and passed through a 0.45 -micron filter. The 5-FU content of the filtrates was determined by reverse-phase high-performance liquid chromatography (Gulliver HPLC System, Jasco Co. Ltd., Tokyo, Japan). Aliquots of the samples were then applied to a column (4.6 ID x $250 \mathrm{~mm}$ ) of Chemcosorb 300-5C18 (Chemco Co. Ltd., Osaka, Japan) under the following chromatographic conditions: monitoring wavelength, $270 \mathrm{~nm}$; flow rate, $1 \mathrm{ml} / \mathrm{min}$; and mobile phase, $20 \mathrm{mM}$ monopotassium phosphate solution ( $\mathrm{pH} 4.5)$. (a)

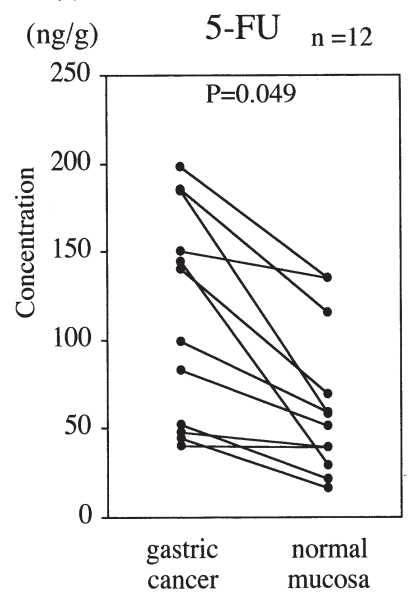

(b)

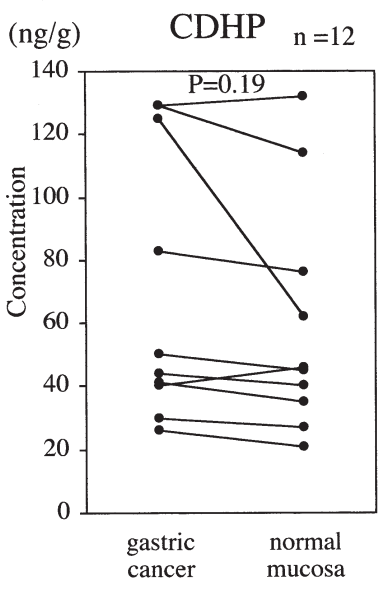

Figure 2. Concentrations of 5-FU and CDHP in gastric cancer tissue and normal gastric mucosa. (a) The 5-FU concentration in gastric cancer tissue was significantly higher than that in normal gastric mucosa $(\mathrm{p}=0.049)$. (b) The CDHP concentration did not differ significantly between gastric cancer tissue and normal mucosa.

Variables. We compared concentrations of 5-FU and CDHP in serum and gastric cancer tissue with those in normal gastric mucosa, and concentrations of 5-FU with those of CDHP in the parietal peritoneum, mesentery, and greater omentum; sites at risk of peritoneal dissemination from gastric cancer. We also examined the correlation between 5-FU and CDHP concentrations in peritoneal tissues and compared 5-FU and CDHP concentrations in the peritoneum and disseminated nodes.

Statistical analysis. The Wilcoxon signed-rank test was used to compare 5-FU and CDHP concentrations in gastric cancer tissue and normal gastric mucosa, and 5-FU and CDHP concentrations among the parietal peritoneum, mesentery, and greater omentum. The Student's t-test was used to 

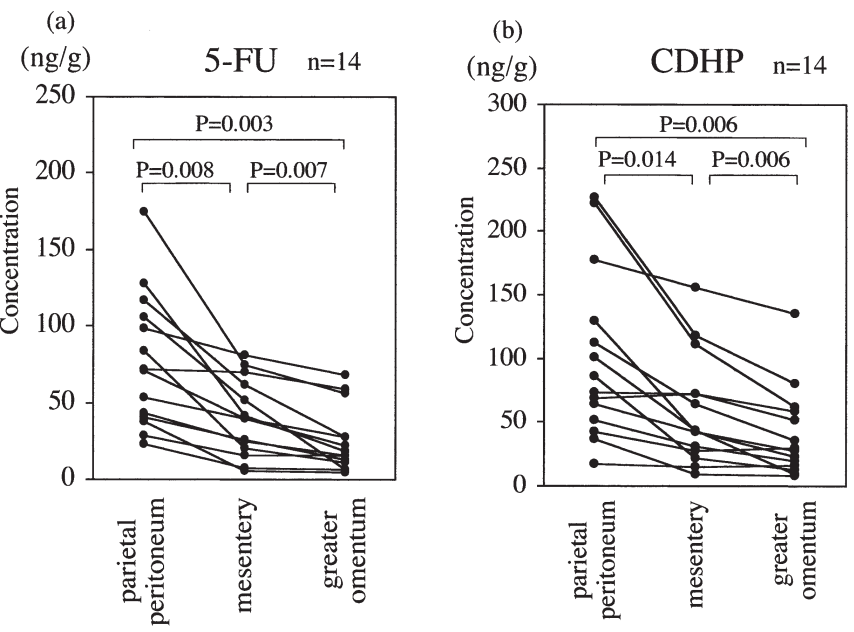

Figure 3. Comparison of 5-FU and CDHP concentrations among the parietal peritoneum, mesentery, and greater omentum. Concentrations of 5-FU (a) and CDHP (b) were highest in the parietal peritoneum, followed by the mesentery and greater omentum; sites at risk for peritoneal dissemination.

compare concentrations of 5-FU or CDHP in the peritoneum and disseminated nodes. A p-value of $<0.05$ was considered significant. The Pearson correlation-coefficient analysis was used to assess the association between 5-FU and CDHP concentrations in peritoneal tissues. The data were analyzed with StatView 5.0.

\section{Results}

Concentrations of 5-FU and CDHP in serum. Serum concentrations of 5-FU and CDHP, $4 \mathrm{~h}$ after the oral administration of TS- 1 were $100.8 \pm 48.5$ and $164.8 \pm 80.7 \mathrm{ng} / \mathrm{ml}$, respectively. The serum 5 -FU concentration was above $50 \mathrm{ng} / \mathrm{ml}$, which was considered the minimal effective level of 5-FU (Fig. 1).

Comparison of 5-FU and CDHP concentrations in gastric cancer tissue and normal gastric mucosa. Concentrations of 5-FU and CDHP were compared in gastric cancer tissue and normal gastric mucosa in 12 patients who underwent gastrectomy. CDHP concentrations did not differ significantly between gastric cancer and normal mucosa. The 5-FU concentration in gastric cancer was significantly higher than

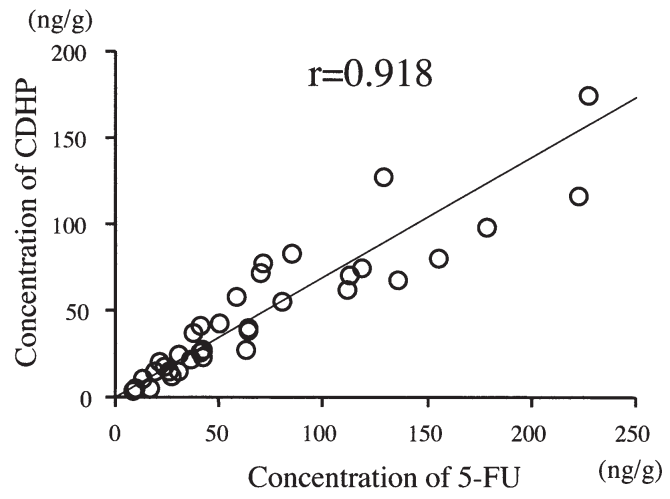

Figure 4. Correlation between 5-FU and CDHP concentrations in the peritoneum. There was a strong correlation between the 5-FU and CDHP concentrations in peritoneal tissue ( $\mathrm{r}=0.918)$.

that in normal mucosa $(\mathrm{p}=0.049)$, suggesting that $5-\mathrm{FU}$ selectively penetrated gastric cancer tissue (Fig. 2).

Comparison of 5-FU and CDHP concentrations among the parietal peritoneum, mesentery, and greater omentum. Concentrations of both 5-FU and CDHP were highest in the parietal peritoneum, followed by the mesentery, and greater omentum. The differences among all three sites were significant for both 5-FU and CDHP concentrations (Fig. 3).

Correlation between 5-FU and CDHP concentrations in the peritoneum. There was a strong correlation between 5-FU and CDHP concentrations in the peritoneum ( $\mathrm{r}=0.918)$, suggesting that the concentration of 5-FU in peritoneal tissues depended on the concentration of CDHP (Fig. 4).

Comparison of 5-FU and CDHP concentrations in the serum and ascites. We measured concentrations of 5-FU and CDHP in 12 patients in whom an adequate volume of ascites for assay was obtained. Concentrations of 5-FU and CDHP in serum were similar to those in the ascites of all patients, suggesting that 5-FU and CDHP linearly passed from serum to ascites (Fig. 5).

Comparison of 5-FU and CDHP concentrations in disseminated nodes and the nearby peritoneum. We measured the concentrations of 5-FU and CDHP in 5 sites of disseminated (a)

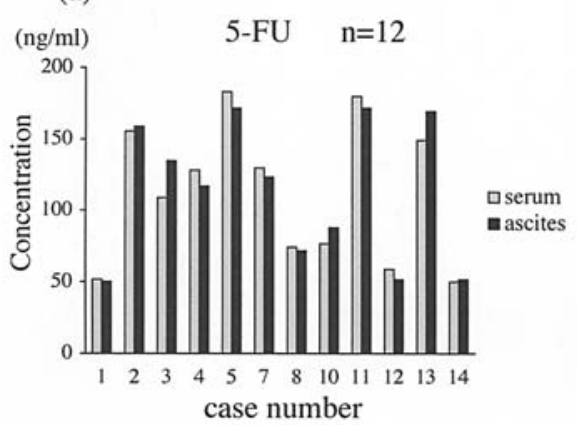

(b)

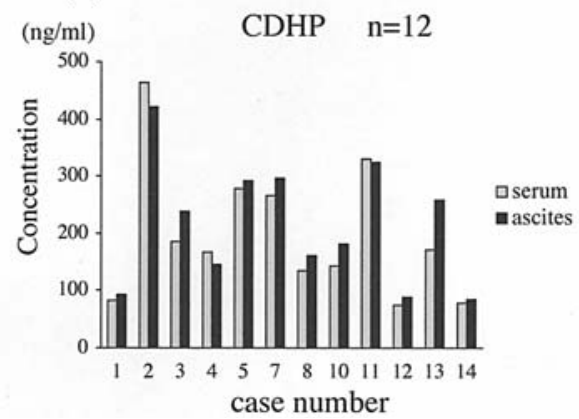

Figure 5. Comparison of 5-FU and CDHP concentrations in the serum and ascites. The concentrations of 5-FU and CDHP in serum were similar to those in the ascites of all patients, suggesting that 5-FU and CDHP linearly passed from serum to ascites. 
(a)

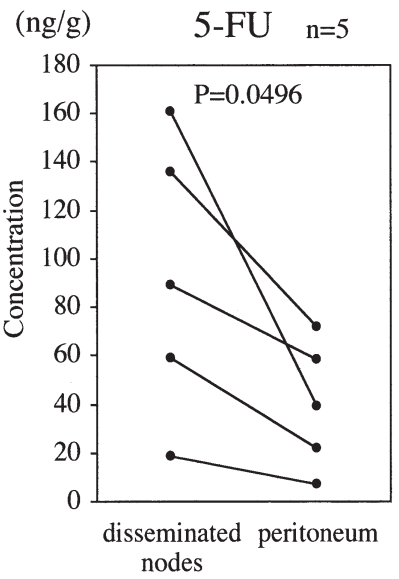

(b)

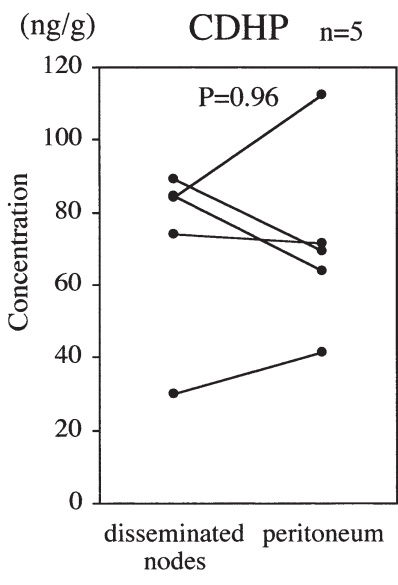

Figure 6. Comparison of 5-FU and CDHP concentrations in disseminated nodes and nearby peritoneum. The concentration of 5-FU was significantly higher in disseminated nodes than in the nearby peritoneum $(\mathrm{p}=0.0496)$ suggesting that 5-FU selectively penetrated disseminated nodes (a).

nodes and the nearby peritoneum in 3 patients in whom disseminated peritoneal nodes were obtained. The CDHP concentration did not differ significantly between disseminated peritoneal nodes and nearby peritoneum. In contrast, the 5-FU concentration was significantly higher in disseminated nodes than in the nearby peritoneum $(\mathrm{p}=0.0496)$, suggesting that 5-FU selectively penetrated disseminated peritoneal nodes (Fig. 6).

\section{Discussion}

Peritoneal dissemination is the most frequent cause of death from gastric cancer, accounting for death in 20-40\% of patients (1). The management of peritoneal dissemination from gastric cancer is thus an important determinant of outcome. Various treatments including systemic chemotherapy (2-5), intraperitoneal chemotherapy (6-8) and thermochemotherapy (9-12) have been attempted.

Yomemura et al reported that combination chemotherapy with cisplatin, mitomycin C, UFT, and etoposide had a high overall response rate $(55 \%)$, but a low response rate for peritoneal dissemination (28\%) (2). That study and a study of combination chemotherapy with etoposide, adriamycin, and cisplatin by Wilke et al showed no improvement in survival with either regimen in patients with peritoneal dissemination (3). A phase II study of sequential methotrexate and 5-FU chemotherapy against peritoneally disseminated gastric cancer had a low response rate (5.7\%) (5). Sugarbaker et al ascribed the poor therapeutic response of peritoneal dissemination to systemic chemotherapy to the bloodperitoneal barrier, consisting of the endothelium, mesentery, and intervening stromal tissue, which separates the cardiovascular system from the peritoneal cavity (13). This barrier negatively affects drug delivery via the bloodstream in patients receiving systemic chemotherapy.

One advantage of intraperitoneally administered anticancer agents was formerly believed to be direct exposure of peritoneal dissemination to high drug concentrations. However, subsequent studies have shown that anticancer

drugs are rapidly absorbed by the peritoneum, resulting in a short duration of active drug concentrations in the peritoneal cavity (14). To solve this problem, Takahashi et al proposed a drug delivery system in which mitomycin $\mathrm{C}$ bound to activated carbon particles is intraperitoneally injected (7).

Techniques for thermochemotherapy have been developed to take advantage of synergistic effects of thermotherapy and chemotherapy against cancer cells. Koga et al reported that continuous hyperthermic peritoneal perfusion (CHPP) with mitomycin $\mathrm{C}$ immediately after surgery improves survival and reduces the rate of recurrence from peritoneal dissemination (9). A randomized, controlled trial by Yonemura et al showed that the rate of survival at 5 years was significantly improved by CHPP (10). However, Hamazoe et al and Kunisaki et al found no significant improvement in the survival rate $(11,12)$. Thus, the therapeutic effectiveness of CHPP remains controversial.

Fujimura et al suggested that subtotal peritoneotomy plus CHPP is significantly more effective than CHPP alone for gastric cancer with peritoneal dissemination (26). However, given the difficult surgical procedure, high risk of complications, and patients' postoperative quality of life, subtotal peritoneotomy plus CHPP falls short of the requirements for standard therapy for gastric cancer with peritoneal dissemination.

Since the late 1990s, several new anticancer drugs have been developed. S-1 was developed as an oral anticancer drug for the treatment of gastric cancer. S-1 consists of tegafur (a prodrug of 5-FU), CDHP (an inhibitor of 5-FU metabolizing enzymes), and oteracil potassium, which specifically reduces gastrointestinal toxicity due to 5-FU, in a molar ratio of 1.0:0.4:1.0 (15). Several studies have reported that $\mathrm{S}-1$ is effective against advanced and recurrent gastric cancer, including cases with peritoneal dissemination (16-22). S-1 is characterized by high therapeutic effectiveness, without compromising patient quality of life. Systemic chemotherapy with conventional anticancer drugs is considered of limited value for the treatment of peritoneal dissemination from gastric cancer because of poor drug penetration of the bloodperitoneal barrier (13). However, S-1 promises to be more effective than conventional systemic chemotherapy against peritoneal dissemination. Yoshikawa et al and Yamagata et al reported that S-1 prolonged survival in a model of gastric cancer with peritoneal dissemination in nude mice $(23,24)$. Those studies also showed high penetration of ascites by 5-FU. We therefore conducted this study to examine the penetration of S-1 to disseminated peritoneal nodes in patients with gastric cancer.

Our study group comprised patients with tumor invasion of the serosa or deeper on preoperative examination, and a high risk of peritoneal dissemination. On the basis of serum concentrations of 5-FU after the oral administration of S-1 and the fact that the peak concentration of 5-FU in tissue was reached approximately $4 \mathrm{~h}$ after treatment, S-1 was given $4 \mathrm{~h}$ before tissue collection on the day of surgery (16).

In our study, the serum 5-FU concentration $4 \mathrm{~h}$ after oral administration of S-1 was maintained at $50 \mu \mathrm{g} / \mathrm{ml}$, the minimal effective concentration of 5-FU. After oral administration of $\mathrm{S}-1$, the peak concentration of 5-FU in serum is reached in approximately $1 \mathrm{~h}$, followed by a gradual decline in the serum 
drug level. Effective serum concentrations of 5-FU are maintained for a longer time after oral S-1 than after other oral 5-FU derivatives because CDHP inhibits 5-FU metabolism by dihydropyrimidine dehydrogenase (DPD) (16).

Comparison of 5-FU and CDHP concentrations between gastric cancer tissue and normal gastric mucosa revealed no significant difference in the concentration of CDHP. In contrast, the concentration of 5-FU was significantly higher in gastric cancer tissue than in normal mucosa, suggesting that 5-FU selectively penetrated gastric cancer tissue and was present in effective concentrations.

Concentrations of 5-FU and CDHP were highest in the parietal peritoneum, followed by the mesentery and greater omentum. The observed differences in drug concentrations might be related to the blood flow distribution and cell density of each type of tissue. There was a strong correlation between 5-FU and CDHP concentrations in peritoneal tissues ( $\mathrm{r}=0.918)$, suggesting that the passage of 5-FU through the peritoneum depends on the concentration of CDHP.

We next examined the passage of 5-FU and CDHP from blood to ascites. Concentrations of 5-FU and CDHP in ascites were similar to those in serum, indicating that 5-FU and CDHP linearly passed from serum to ascites via the peritoneum. Thus, the theory that the delivery of systemically administered anticancer agents is negatively affected by the blood-peritoneal barrier does not apply to $S-1$. After the administration of S-1, 5-FU and CDHP directly pass from blood to ascites through the peritoneum, acting as a 'sieve.' After systemic administration of conventional 5-FU derivatives, 5-FU is metabolized by DPD in peritoneal tissues on passage from capillaries supplying the peritoneum to the peritoneal cavity. In contrast, after oral administration of S-1, CDHP passes through the peritoneum with 5-FU, inhibiting its metabolism. Both 5-FU and CDHP are linearly transferred to the ascites. This mechanism may contribute to the effectiveness of S-1 for peritoneal dissemination. Intraperitoneally administered anticancer drugs are rapidly absorbed, resulting in a low drug concentration in the peritoneal cavity. However, previous studies showing prolonged effective concentrations of 5-FU and CDHP in serum after oral S-1 (16), and our results demonstrating similar concentrations of 5-FU and CDHP in serum and ascites, suggest that high intraperitoneal concentrations of 5-FU are maintained for a prolonged time after the oral administration of S-1.

When we compared 5-FU and CDHP concentrations between disseminated nodes and the nearby peritoneum, the 5-FU concentration was significantly higher in disseminated nodes than in nearby peritoneum, whereas the CDHP concentration did not differ. This may be attributed to the fact that the metabolism of 5-FU, which has a high affinity for gastric cancer cells, was inhibited by CDHP uptake in disseminated nodes, resulting in prolonged, high concentrations of 5-FU in disseminated nodes.

In conclusion, our results show that 5-FU in blood linearly passes through the peritoneum and enters ascites after oral administration of S-1 to patients who have gastric cancer with peritoneal dissemination. CDHP-induced inhibition of 5-FU metabolism by DPD has an important role in the delivery of 5-FU to peritoneal tissues. High concentrations of 5-FU are selectively transferred to peritoneal disseminated cells, contributing to the effectiveness of S-1 for gastric cancer with peritoneal dissemination.

\section{References}

1. Yonemura Y, Bandou E, Kinoshita K, et al: Effective therapy for peritoneal dissemination in gastric cancer. Surg Oncol Clin N Am 12: 635-648, 2003.

2. Yonemura Y and Miyazaki I: Presence and future in neoadjuvant chemotherapy against gastric cancer. Gan To Kagaku Ryoho 22: 1893-1904, 1995.

3. Wilke H: Management of gastric cancer. Sugarbaker P (ed). Kluwer Academic Publishers, Boston, pp363-373, 1991.

4. Tahara M, Ohtsu A, Boku N, et al: Sequential methotrexate and 5-fluorouracil therapy for gastric cancer patients with peritoneal dissemination: a retrospective study. Gastric Cancer 4: 212-218, 2001.

5. Yamao T, Shimada Y, Shirao K, et al: Phase II study of sequential methotrexate and 5-fluorouracil chemotherapy against peritoneally disseminated gastric cancer with malignant ascites: a report from the Gastrointestinal Oncology Study Group of the Japan Clinical Oncology Group, JCOG 9603 Trial. Jpn J Clin Oncol 34: 316-322, 2004.

6. Markman M: Intraperitoneal chemotherapy: Semin Oncol 18: 248-254, 1991.

7. Takahashi T, Hagiwara A, Shimotsuma M, Sawai K and Yamaguchi T: Prophylaxis and treatment of peritoneal carcinomatosis: intraperitoneal chemotherapy with mitomycin C bound to activated carbon particles. World J Surg19: 565-569, 1995.

8. Jeung HC, Rha SY, Jang WI, Noh SH and Chung HC: Treatment of advanced gastric cancer by palliative gastrectomy, cytoreductive therapy and postoperative intraperitoneal chemotherapy. Br J Surg 89: 460-466, 2002.

9. Koga S, Hamazoe R, Maeta M, Shimizu N, Murakami A and Wakatsuki T: Prophylactic therapy for peritoneal recurrence of gastric cancer by continuous hyperthermic peritoneal perfusion with mitomycin C. Cancer 61: 232-237, 1988.

10. Yonemura Y, de Aretxabala X, Fujimura T, et al: Intraoperative chemohyperthermic peritoneal perfusion as an adjuvant to gastric cancer: final results of a randomized controlled study. Hepatogastroenterology 48: 1776-1782, 2001.

11. Hamazoe R, Maeta M and Kaibara N: Intraperitoneal thermochemotherapy for prevention of peritoneal recurrence of gastric cancer. Final results of a randomized controlled study. Cancer 73: 2048-2052, 1994.

12. Kunisaki C, Shimada H, Nomura M, Akiyama H, Takahashi M and Matsuda G: Lack of efficacy of prophylactic continuous hyperthermic peritoneal perfusion on subsequent peritoneal recurrence and survival in patients with advanced gastric cancer. Surgery 131: 521-528, 2002.

13. Sugarbaker PH, Stuart OA, Vidal-Jove J, Pessagno AM and DeBruijn EA: Pharmacokinetics of the peritoneal-plasma barrier after systemic mitomycin $\mathrm{C}$ administration. Cancer Treat Res 82: 41-52, 1996

14. Aznavoorian S, Murphy AN, Stetler-Stevenson WG and Liotta LA: Molecular aspects of tumor cell invasion and metastasis. Cancer 71: 1368-1383, 1993.

15. Shirasaka T, Nakano K, Takechi T, et al: Antitumor activity of $1 \mathrm{M}$ potassium oxonate (S-1) against human colon carcinoma orthotopically implanted into nude rats. Cancer Res 56: 2602-2606, 1996.

16. Sakata Y, Ohtsu A, Horikoshi N, Sugimachi K, Mitachi Y and Taguchi T: Late phase II study of novel oral fluoropyrimidine anticancer drug S-1 (1 M tegafur, 0.4 M gimestat, $1 \mathrm{M}$ otastat potassium) in advanced gastric cancer patients. Eur J Cancer 34: 1715-1720, 1998.

17. Sugimachi K, Maehara Y, Horikoshi N, et al: An early phase II study of oral S-1, a newly developed 5-fluorouracil derivative for advanced and recurrent gastrointestinal cancers. The S-1 Gastrointestinal Cancer Study Group. Oncology 57: 202-210, 1999.

18. Koizumi W, Kurihara M, Nakano S and Hasegawa K: Phase II study of S-1, a novel oral derivative of 5-fluorouracil, in advanced gastric cancer. For the S-1 Cooperative Gastric Cancer Study Group. Oncology 58: 191-197, 2000.

19. Kobayashi O, Konishi K, Kanari M, et al: Unusual survival for more than 2 years with peritoneal metastases of gastric cancer. Gastric Cancer 5: 47-50, 2002. 
20. Osugi H, Takada N, Takemura M, et al: Oral fluoropyrimidine anticancer drug TS-1 for gastric cancer patients with peritoneal dissemination. Oncol Rep 9: 811-815, 2002.

21. Fujitani K, Tsujinaka T and Hirao M: Feasibility study of S-1 for respectable gastric cancer with peritoneal seeding. Hepatogastroenterology 50: 889-892, 2003.

22. Kitamura Y, Hayashi K, Sasagawa T, Oguma $\mathrm{H}$ and Takasaki K: Pilot study of S-1 in patients with disseminated gastric cancer. Drugs Exp Clin Res 29: 125-130, 2003.

23. Yoshikawa T, Yanoma S, Tsuburaya A, et al: Effects of TS-1 on peritoneal dissemination of gastric cancer in nude mice. Hepatogastroenterology 51: 1554-1557, 2004.
24. Yamagata S, Nakata B and Hirakawa K: Dihydropyrimidine dehydrogenase inhibitory fluoropyrimidine S-1 may be effective against peritoneal dissemination in gastric cancer. Oncol Rep 12: 973-978, 2004.

25. Japanese Research Society for Gastric Cancer. Japanese Classification of Gastric Carcinoma. First English Edition. Kanehara and Co., Ltd, Tokyo, 1995.

26. Fujimura T, Yonemura Y, Nakagawara H, et al: Subtotal peritonectomy with chemohyperthermic peritoneal perfusion for peritonitis carcinomatosa in gastrointestinal cancer. Oncol Rep 7: 809-814, 2000. 